NOUVELLE

\section{Comment limiter la progression des tumeurs prostatiques déficientes en PTEN?}

Élise Grelet ${ }^{1}$, Maxime Parisotto ${ }^{1,2}$, Daniel Metzger ${ }^{1}$
${ }^{1}$ Institut de génétique et de biologie moléculaire et cellulaire (IGBMC), CNRS UMR7104, Inserm U1251, université de Strasbourg, 1 rue Laurent Fries, 67400 Illkirch, France.

${ }^{2}$ Centre de recherche du centre hospitalier de l'université de Montréal (CRCHUM), 900, rue Saint-Denis, Montréal (Québec) H2X 0A9, Canada.

daniel.metzger@unistra.fr
> Le cancer de la prostate est le cancer masculin le plus fréquent et la deuxième cause de mortalité par cancer dans les pays industrialisés [1]. La progression des tumeurs de l'épithélium glandulaire de la prostate est bien caractérisée histologiquement. À l'origine, des néoplasies prostatiques intra-épithéliales (PIN) apparaissent et certaines évoluent, après plusieurs décennies (phase de latence), en adénocarcinomes localement invasifs. Ces derniers, lorsqu'ils progressent en cancers métastatiques, conduisent généralement au décès des patients. Environ $40 \%$ des hommes de plus de 50 ans développent des PIN en majorité indolentes, mais $1 \%$ des patients développent des tumeurs cliniquement significatives. Détecté à un stade local, le cancer de la prostate est éradiqué dans 70 à $80 \%$ des cas par la prostatectomie, la radiothérapie ou la cryothérapie. Les stades plus avancés sont traités principalement avec des anti-androgènes. Ces derniers induisent généralement la régression des tumeurs, mais, après un temps de latence variable, des rechutes sont observées chez de nombreux patients en raison de l'apparition de résistances à ces thérapies [2]. Une meilleure connaissance des mécanismes moléculaires à l'origine de l'agressivité des tumeurs de la prostate et l'identification de nouvelles cibles thérapeutiques sont donc nécessaires afin de développer des thérapies qui soient efficaces.

PTEN (phosphatase and tensin homolog deleted on chromosome 10) est le gène suppresseur de tumeur le plus souvent muté dans les cancers de la prostate.
C'est un régulateur négatif de la voie PI3K (phosphoinositide 3-kinase)/Akt (protein kinase $B$ ) et sa délétion stimule la prolifération et la survie cellulaire. La perte de PTEN est fortement corrélée à une plus grande agressivité des tumeurs prostatiques, avec l'apparition de métastases et une plus grande probabilité d'échappement aux antiandrogènes. Elle est donc associée à un pronostic défavorable. Dans les cellules épithéliales prostatiques murines, l'absence du gène induit la formation de PIN $[3,4]$. La progression de ces néoplasies en adénocarcinomes est limitée par une entrée en sénescence des cellules épithéliales prostatiques [5] qui, selon cette étude, n'était pas consécutive à une phase d'hyperprolifération, ni a un stress réplicatif ou à une réponse à des dommages de I'ADN. Ces résultats vont à l'encontre des caractéristiques connues de la sénescence induite par les oncogènes (SIO) [6] $(\rightarrow)$. Un nouveau

$\rightarrow$ Voir la Synthèse de $\varepsilon$. Goy et C. Abbadie, $\mathrm{m} / \mathrm{s} \mathrm{n}^{\circ} 3$, mars 2018, page 223

type de sénescence associée à la perte de PTEN et nommé PTEN loss-induced senescence (PICS) a donc été proposé [5]. Par ailleurs, la sécrétion d'IL-IRA (interleukine-1 receptor antagonist) par un infiltrat de cellules myéloïdes GRl-positives ${ }^{1}$, permettant à certaines cellules épithéliales prostatiques déficientes en PTEN de ne pas entrer en sénescence et de continuer à proliférer, contribue à la formation des PIN [7].

${ }^{1}$ GRI est un antigène présent sur les cellules myéloïdes Ly6C et Ly6G.
Afin de mieux caractériser l'impact de la perte de PTEN dans les cellules épithéliales prostatiques, nous avons généré et étudié des souris mutantes (PTEN ${ }^{(i) p e-/-}$ ) chez lesquelles le gène est sélectivement invalidé dans les cellules épithéliales prostatiques après la puberté. Nos résultats montrent que la perte de PTEN entraîne la phosphorylation d'Akt (Figure IA et B), et que la prolifération des cellules épithéliales prostatiques augmente 1 mois après la perte du gène pour atteindre un pic après 3 mois (Figure $I C$ et $D$ ). Elle diminue ensuite fortement après 6 mois. Entre 5 et 12 mois, la majorité des cellules constituant les PIN sont sénescentes (Figure 1H) et produisent de nombreuses cytokines et chimiokines. Les PIN sont alors infiltrées par des cellules myéloïdes positives pour GR-1, qui ne sont détectées qu'après 2 mois, tout comme les marqueurs de sénescence [8]. Nos résultats montrent donc que la perte de PTEN dans les cellules épithéliales prostatiques est responsable de la prolifération active des cellules, avant l'entrée en sénescence et indépendamment des cellules positives pour GR-1. Des foyers nucléaires de $\gamma H 2 A X$ (la forme phosphorylée de l'histone $\mathrm{H} 2 \mathrm{AX}$ ) sont également observés dans de nombreuses cellules épithéliales prostatiques déficientes pour PTEN (Figure IF), indiquant l'activation d'une réponse aux dommages de l'ADN dans ces cellules [9]. Elles présentent aussi plusieurs marqueurs de stress réplicatif tels que RPA32, RPA32 S4/S8 et ATR ${ }^{2}$.

${ }^{2}$ RPA42 : protéine réplicative de $42 \mathrm{KD}$ a phosphorylée sur les sérines 4/8 (RPA32 S4/S8) au cours du stress réplicatif ; ATR : ataxia telangiectasia and Rad3-related protein. 


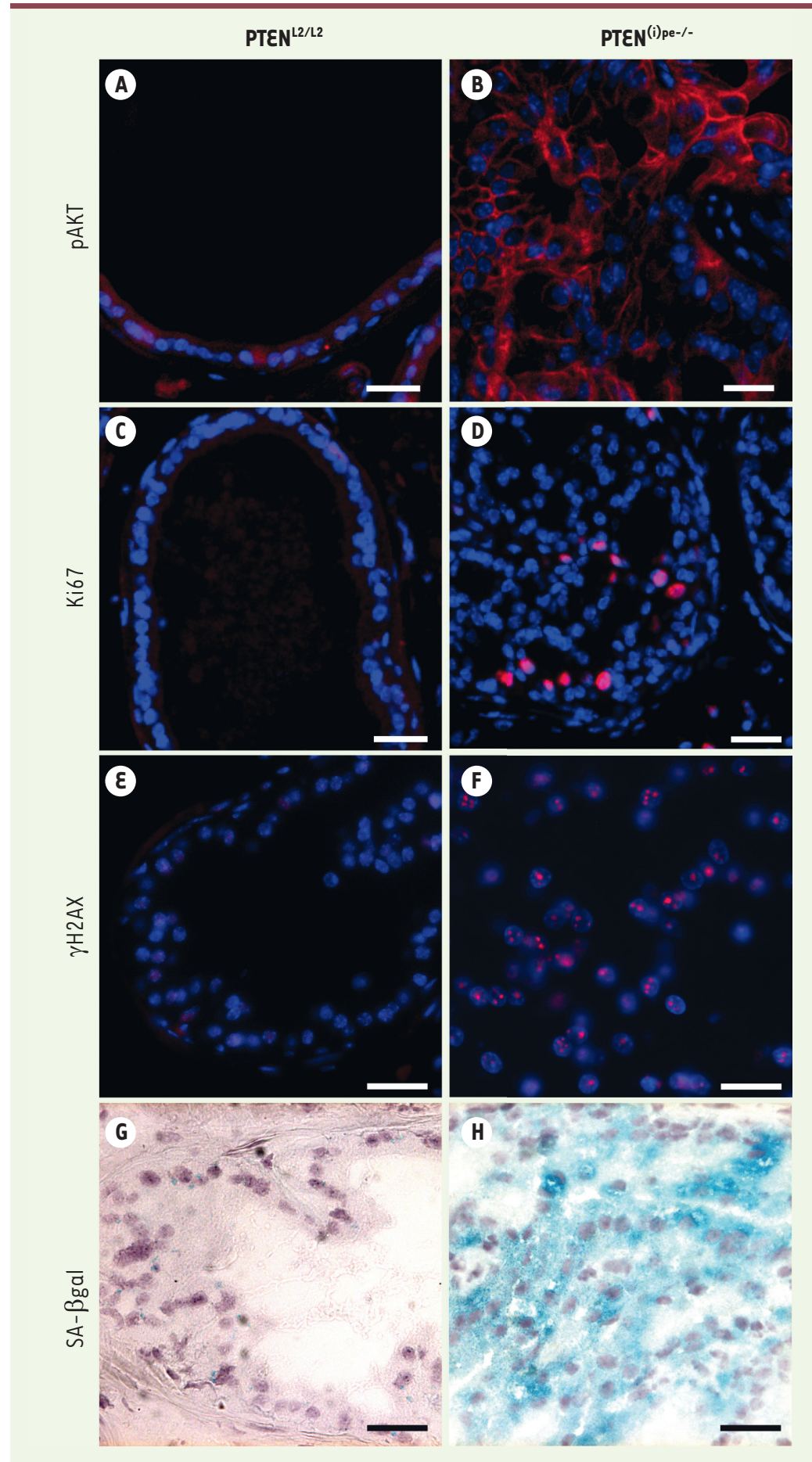

Figure 1. Caractérisation des néoplasies prostatiques intra-épithéliales induites par invalidation conditionnelle de PTEN chez la souris. Comparaison de l'expression sur des coupes de prostate des marqueurs pAkt (phospho-protein kinase $B$ ) en $A$ et $B$, Ki67 (marqueur de prolifération) en $C$ et $D$ et $\gamma \mathrm{H} 2 \mathrm{AX}$ (phosphorylation de l'histone H2AX induite suite à des dommages de l'ADN) en $\varepsilon$ et $F$ chez des souris âgées de 5 mois PTEN ${ }^{\mathrm{L} 2 / L 2}$ sauvages pour l'expression de PTEN ou PTEN ${ }^{(i) p e-/-}$ chez lesquelles le gène PTEN est sélectivement invalidé dans les cellules épithéliales prostatiques après la puberté. Les cellules épithéliales sénescentes de souris PTEN(i)pe-/- âgées de 7 mois expriment la $\beta$-galactosidase (SA- $\beta$-gal pour senescence-associated $\beta$-galactosidase), marquage bleu $(H)$, contrairement aux cellules épithéliales prostatiques de souris PTEN ${ }^{L 2 / L 2}(G)$.
Les mécanismes aboutissant à la PICS sont donc semblables à ceux de la sénescence induite par les oncogènes. Les stratégies thérapeutiques visant son induction présentent donc des risques, car celles-ci entraîneraient un stress réplicatif, des dommages à l'ADN et potentiellement la mutation d'autres gènes. Ces mutations, ainsi que la sécrétion de cytokines et de chimiokines, sont susceptibles de favoriser l'échappement de la sénescence et ainsi contribuer au développement tumoral.

Dans les cellules épithéliales prostatiques déficientes pour PTEN, l'activation d'une réponse aux dommages de I'ADN par le stress réplicatif induit une stabilisation du suppresseur de tumeurs p53 (Figure 2). Cependant, à des temps précoces après la perte de PTEN, p53 est régulée négativement par $\mathrm{Mdm} 2$ (mouse double minute 2 homolog), potentiellement via sa phosphorylation par Akt qui augmente son activité $\varepsilon 3$-ubiquitineligase pour p53. La caséine kinase I (CKI) semble être un acteur important dans la stabilisation progressive de $p 53$. Son expression est induite par la réponse aux dommages à l'ADN, et elle est responsable de la diminution de l'interaction entre Mdm2 et p53 et/ou de la promotion de la dégradation de Mdm2 [10, 11]. Les niveaux de transcrits de CKI sont augmentés entre 3 et 5 mois après I'invalidation de PTEN (Figure 2). À noter que l'invalidation de $\mathrm{p} 53$ dans les cellules épithéliales prostatiques déficientes en PTEN permet de lever la barrière de sénescence, accélère le développement d'adénocarcinomes et favorise l'apparition de tumeurs sarcomatoïdes métastatiques [8, 12] (et données non publiées). Nos travaux soulignent l'importance de l'utilisation de modèles pertinents pour l'étude de pathologies. In vivo, la possibilité de contrôler l'invalidation du gène PTEN après la puberté, sélectivement dans les cellules épithéliales luminales prostatiques (grâce à la technologie $\left(r e \varepsilon R^{T 2}\right)^{3}$, est en effet

${ }^{3}$ Recombinase Cre chimérique dont l'activité est inductible par le tamoxifène. 


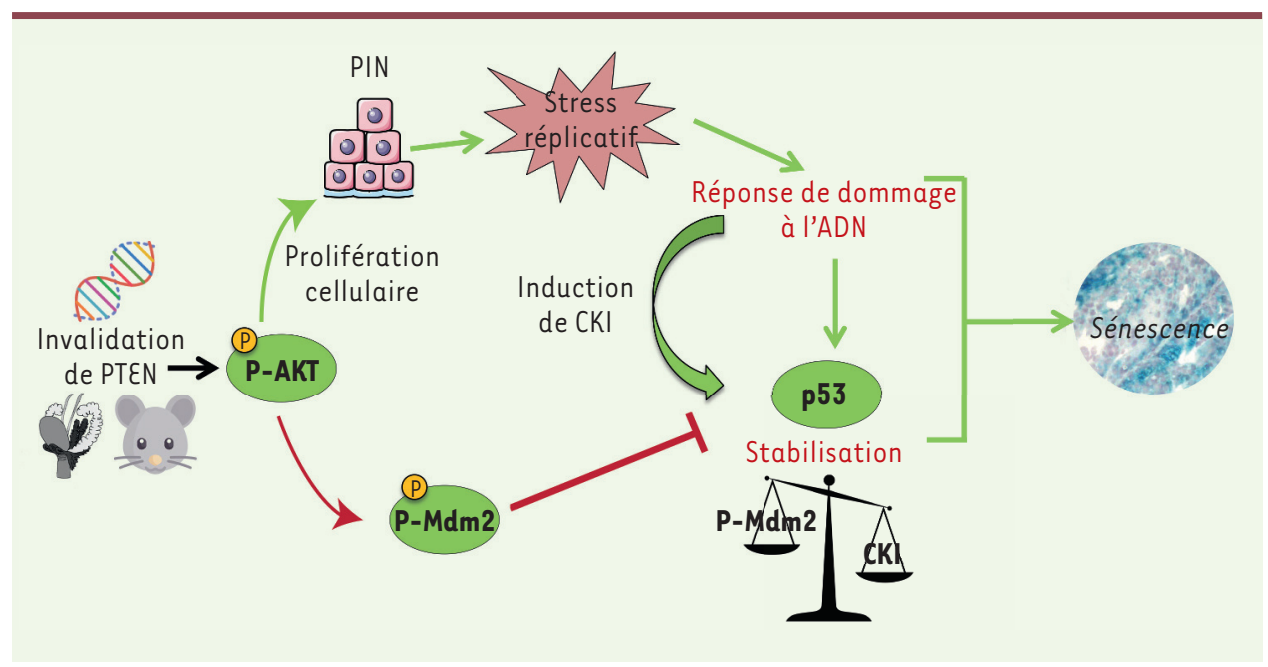

Figure 2. Signalisation impliquée dans la formation de néoplasies prostatiques intra-épithéliales sénescentes. Chez les souris déficientes en PTEN, une augmentation de la phosphorylation d'Akt (protein kinase $B$ ) induit la prolifération des cellules et provoque des néoplasies prostatiques intra-épithéliales (PIN). À des temps précoces après la perte de PTEN, p53 est régulée négativement par Mdm2 (mouse double minute 2 homolog), potentiellement via sa phosphorylation par Akt qui augmente son activité $\varepsilon 3$ ubiquitine-ligase envers p53. Progressivement, p53 est stabilisée via l'activation d'une réponse aux dommages de l'ADN par le stress réplicatif et l'augmentation de l'expression de la caséine kinase I (CKI). La progression des cellules épithéliales prostatiques en adénocarcinome est limitée par leur entrée en sénescence.

un avantage majeur par rapport aux autres modèles murins de cancer de la prostate dans lesquels PTEN est invalidé dans les cellules épithéliales de la prostate immature. En outre, les cultures cellulaires in vitro sont exposées à de nombreux facteurs de croissance et à une forte pression partielle d'oxygène, qui induisent un stress cellulaire, ce qui pourrait favoriser l'entrée en sénescence des cellules [12]. L'extrapolation des données obtenues in vitro, notamment celles relatives à la sénescence cellulaire, à des situations in vivo doit donc être réalisée avec beaucoup de prudence.

Notre étude apporte donc un nouveau regard sur la phase latente du cancer de la prostate et ouvre de nouvelles perspectives thérapeutiques. Nos résultats montrent en effet que la perte de PTEN dans les cellules épithéliales prostatiques provoque une réponse de dommage de I'ADN induite par un stress réplicatif et une entrée très progressive des cellules en sénescence. L'hyperactivation d'Akt, causée par la perte de PTEN, est probablement responsable de la dégradation de p53 par Mdm2, et donc du retard d'entrée en sénescence avant que les niveaux de CKI soient suffisants pour induire la stabilisation et l'activation de p53. Bien qu'il soit possible que des inhibiteurs d'Akt ou de l'interaction entre Mdm2 et p53 favorisent l'entrée en sénescence et ainsi augmentent la durée de la phase de latence, cibler et éradiquer les cellules épithéliales prostatiques déficientes en PTEN représente une alternative thérapeutique pour prévenir la progression tumorale prostatique. $\diamond$

How to limit the progression of PTENdeficient prostatic tumors?

\section{REMERCIEMENTS}

Nous remercions le CNRS, l'Inserm, l'université de Strasbourg, la Fondation ARC pour la recherche sur le cancer, la ligue contre le cancer, l'association Alsace contre le cancer, l'association pour la recherche sur les tumeurs de la prostate, le centre d'ingénierie moléculaire européen, l'agence nationale de la recherche (ANR-10LABX-0030-INRT et programme investissements d'avenir IANR-10-IDEX-0002-02). MP a bénéficié d'une bourse de l'association pour la recherche à l'IGBMC (ARI) et $\varepsilon G$ d'une bourse du Ministère de l'enseignement supérieur et de la recherche.

\section{LIENS D'INTÉRÊT}

Les auteurs déclarent n'avoir aucun lien d'intérêt concernant les données publiées dans cet article.

\section{RÉFÉRENCES}

1. Siegel RL, Miller KD, Jemal A. Cancer statistics, 2018. CA CancerJ Clin $2018 ; 68: 7-30$.

2. Shen MM, Abate-Shen C. Molecular genetics of prostate cancer: new prospects for old challenges. Genes Dev 2010 ; 24 : 1967-2000.

3. Chen Z, Trotman LC, Shaffer D, et al. Crucial role of p53-dependent cellular senescence in suppression of Pten-deficient tumorigenesis. Nature 2005 ; 436 : 725-30.

4. Ratnacaram CK, Teletin M, Jiang M, et al. Temporally controlled ablation of PTEN in adult mouse prostate epithelium generates a model of invasive prostatic adenocarcinoma. Proc Natl Acad Sci USA 2008 ; 105 : 2521-6.

5. Alimonti A, Nardella C, Chen Z, et al. A novel type of cellular senescence that can be enhanced in mouse models and human tumor xenografts to suppress prostate tumorigenesis. J Clin Invest 2010 ; 120 : 68193.

6. Goy $\varepsilon$, Abbadie C. Sénescence et cancer Double jeu. Med Sci (Paris) 2018 ; $34: 223-30$.

7. Di Mitri D, Toso A, Chen JJ, et al. Tumour-infiltrating $\mathrm{Gr}-\mathrm{I}^{+}$myeloid cells antagonize senescence in cancer. Nature 2014 ; 515 : 134-7.

8. Parisotto $M$, Grelet $\varepsilon$, દl Bizri R, et al. PTEN deletion in luminal cells of mature prostate induces replication stress and senescence in vivo. J Exp Med $2018 ; 215$ : 1749-63.

9. Mah LJ, El-Osta A, Karagiannis TC. GammaH2AX: a sensitive molecular marker of DNA damage and repair. Leukemia $2010 ; 24: 679-86$.

10. Inuzuka H, Tseng A, Gao D, et al. Phosphorylation by casein kinase I promotes the turnover of the Mdm2 oncoprotein via the SCF(beta-TRCP) ubiquitin ligase. Cancer Cell $2010 ; 18$ : 147-59.

11. Knippschild U, Kruger M, Richter J, et al. The CKI family: contribution to cellular stress response and its role in carcinogenesis. Front Oncol 2014 ; $4: 96$.

12. Serrano M, Blasco MA. Putting the stress on senescence. Curr Opin Cell Biol 2001 ; 13 : 748-53. 